\title{
Statistical moments for classical and quantum dynamics: formalism and generalized uncertainty relations
}

\author{
David Brizuela* \\ Fisika Teorikoa eta Zientziaren Historia Saila, UPV/EHU, 644 P.K., 48080 Bilbao, Spain and \\ Institut für Theoretische Physik, Universität zu Köln, Zülpicher Straße 77, 50937 Köln, Germany
}

\begin{abstract}
The classical and quantum evolution of a generic probability distribution is analyzed. To that end, a formalism based on the decomposition of the distribution in terms of its statistical moments is used, which makes explicit the differences between the classical and quantum dynamics. In particular, there are two different sources of quantum effects. Distributional effects, which are also present in the classical evolution of an extended distribution, are due to the fact that all moments can not be vanishing because of the Heisenberg uncertainty principle. In addition, the non-commutativity of the basic quantum operators add some terms to the quantum equations of motion that explicitly depend on the Planck constant and are not present in the classical setting. These are thus purely-quantum effects. Some particular Hamiltonians are analyzed that have very special properties regarding the evolution they generate in the classical and quantum sector. In addition, a large class of inequalities obeyed by high-order statistical moments, and in particular uncertainty relations that bound the information that is possible to obtain from a quantum system, are derived.
\end{abstract}

PACS numbers: 03.65.-w, 03.65.Sq, 98.80.Qc

\section{INTRODUCTION}

The classical limit of quantum mechanics is very subtle. One of the main problems to define such a limit is the difference between the formalisms used to describe these theories. Quantum mechanics is analytical since it is based on Hilbert spaces and operators acting thereon, whereas classical mechanics is geometrical: it is defined on symplectic manifolds and with Hamiltonian vector fields describing the evolution. In addition, apart from the purely quantum and purely classical theories, there are also different attempts to construct hybrid theories that consider a direct coupling between classical and quantum degrees of freedom, see e.g. [1 $[-6]$. Nevertheless, many of these theories suffer from several drawbacks [7] and will not be considered here; see Ref. [8] for a discussion on these hybrid theories within a formalism similar to the one presented in this paper.

The intuitive idea one usually has in mind about a semiclassical state is that of a peaked coherent state, whose centroid (the coordinates on the phase space of the expectation value of the position and momentum operators) follows a classical orbit in phase space. Nonetheless, in Ref. [9] a different idea was presented: the classical limit of a quantum system is not a single classical orbit, but an ensemble of orbits. Therefore, even if it is not peaked and its centroid does not follow a classical trajectory, a quantum state can behave essentially classically if it follows the evolution given by the Liouville equation for an equivalent classical ensemble. In particular it is well known that generically the centroid of a quantum state does not follow a classical trajectory. But this very

*Electronic address: david.brizuela@ehu.es same thing happens with the evolution of the centroid of a classical distribution; so such an effect can not be regarded as completely quantum.

Another reason to work with classical probability distributions is that, even if in the context of classical mechanics in principle one could have a point in the phase space as initial condition, in practice there are always measurement errors that introduce some uncertainty on the knowledge about the initial state. Hence, this issue forces us to consider also the evolution of extended probability distributions on a purely classical setting.

The difference between a quantum and classical distributional evolution of an initial state could be of particular importance in the context of quantum cosmology. Leaving aside theories that involve multiverses, there is only one realization of the universe and thus one dynamical state that describes it. If, at some point, we were able to measure some properties of that state, it will be necessary to compare its classical and quantum evolution in order to know if the universe is indeed behaving quantum mechanically or this distributional behavior is just due to the error on our measurements.

As will be made explicit in this paper, a formalism very well suited to compare the classical and quantum evolutions of a given physical system is the one developed in [10] for Hamiltonians corresponding to a particle on a potential. In that paper a decomposition of the wave function into its infinite set of statistical moments is considered. The moments contain the same physical information as the wave function, but have the great advantage of being observable. The evolution equations of these variables give rise to the Hamilton equations for the expectation values of the position and momentum operators, with corrections coming from the moment variables. On the other hand, in the same way as for the quantum theory, it is possible to define the moments corresponding 
to a classical ensemble and obtain their evolution equations. In this way, the classical and quantum evolution of a given physical system is described in a very similar setting and the comparison between them is straightforward.

A similar formalism to the one developed in [10], but with a different ordering of the basic variables, was presented in [11] for generic Hamiltonians in a canonical framework. This formalism has been widely applied to different cosmological models (see [12] for a recent review). One of the advantages of this approach is that it can be applied also when the dynamics is described by a Hamiltonian constraint, as opposed to a Hamiltonian function [13]. In this context, isotropic cosmological models with negative and positive cosmological constant have been studied in Ref. [14] and [15] respectively. It has also been applied to simple bounce scenarios in loop quantum cosmology [16]. Furthermore, the problem of time in semiclassical regimes, as well as the relational quantum dynamics, has also been considered in [17, 18].

In the present paper the classical counterpart to the quantum formalism developed in [11] will be presented. In this way, a formalism similar to the one introduced in [10] will be obtained, but valid for generic Hamiltonians and with a different ordering of the basic variables. In addition, due to the generic formula between two moments obtained in [15, 19], much more compact evolution equations will be presented, which allow for an easier understanding of the terms involved. Harmonic and linear Hamiltonians will be considered due to their special properties regarding the classical and quantum evolution they generate. Furthermore, making use of the CauchySchwarz inequality, high-order inequalities obeyed by statistical moments will be systematically obtained. In the quantum case, a specific subset of these inequalities will give rise to high-order uncertainty relations, that generalize the well-known Heisenberg uncertainty principle.

The rest of the article is organized as follows. In Sec. II the general formalism is explained. In Subsec. IA a brief summary of the quantum formalism introduced in [11] is given, whereas in Subsec. [IB its classical analog is developed. The rest of this section underlines the differences between the classical and quantum settings, and analyzes how to obtain dynamical and stationary states in this context. Section 【II discusses the special classical and quantum behavior of harmonic and linear Hamiltonians. In Sec. IV high-order inequalities obeyed by both classical and quantum statistical moments are obtained and analyzed. Finally, Sec. V presents the conclusions and summarizes the main results of the article.

\section{GENERAL FORMALISM}

\section{A. Quantum moments}

In this subsection the formalism developed in [11] is briefly reviewed. Let us assume a quantum mechanical system with one degree of freedom described by the basic conjugate operators $(\hat{q}, \hat{p})$. We define the quantum moments

$$
G^{a, b}:=\left\langle(\hat{p}-p)^{a}(\hat{q}-q)^{b}\right\rangle_{\mathrm{Weyl}},
$$

where $p:=\langle\hat{p}\rangle, q:=\langle\hat{q}\rangle$ are the expectation values of the momentum and position operator, respectively, and the subscript Weyl stands for totally symmetric ordering. The sum between its two indices $(a+b)$ will be referred as the order the moment $G^{a, b}$.

Note that through this decomposition the wave function $\Psi(q, t)$ gets replaced by its infinite set of statistical moments $G^{a, b}(t)$, which only depend on time. This is quite similar to what is done in physical problems with certain symmetry: use special functions adapted to that symmetry and remove the dependence on the trivial directions, like for example spherical harmonics when dealing with spherical symmetry.

Performing the Taylor expansion of the Hamiltonian operator $\hat{H}$, an effective Hamiltonian $H_{Q}$ is obtained as function of the expectation values and moments:

$$
\begin{aligned}
H_{Q}\left(q, p, G^{a, b}\right) & =\langle\hat{H}(\hat{q}, \hat{p})\rangle_{\mathrm{Weyl}} \\
& =\langle\hat{H}(\hat{q}-q+q, \hat{p}-p+p)\rangle_{\mathrm{Weyl}} \\
& =\sum_{a=0}^{\infty} \sum_{b=0}^{\infty} \frac{1}{a ! b !} \frac{\partial^{a+b} H}{\partial p^{a} \partial q^{b}} G^{a, b} \\
& =H(q, p)+\sum_{a+b \geq 2} \frac{1}{a ! b !} \frac{\partial^{a+b} H}{\partial p^{a} \partial q^{b}} G^{a, b}
\end{aligned}
$$

The classical Hamiltonian $H(q, p)$ is obtained by replacing $\hat{q}$ and $\hat{p}$ by their corresponding expectation values $q$ and $p$, respectively, in the explicit expression of the Hamiltonian operator $\hat{H}(\hat{q}, \hat{p})$. It can be shown that, if one defines the Poisson bracket for expectation values of arbitrary operators $\hat{f}$ and $\hat{g}$ by the relation $\{\langle\hat{f}\rangle,\langle\hat{g}\rangle\}=$ $-i \hbar^{-1}\langle[\hat{f}, \hat{g}]\rangle$, the evolution generated by this Hamiltonian is equivalent to the Schrödinger flow of quantum states. Thus, this effective Hamiltonian encodes the complete dynamical information of our variables: the expectation values $(q, p)$ and the infinite set of moments. With the mentioned definition, it is immediate to see that, given the commutation relation $[\hat{q}, \hat{p}]=i \hbar$, the Poisson bracket between conjugate variables reduces to the canonical one: $\{q, p\}=1$. Moreover, one can easily show that moments have vanishing Poisson brackets with the basic expectation values:

$$
\left\{G^{a, b}, p\right\}=0=\left\{G^{a, b}, q\right\} .
$$

The general Poisson brackets between moments is more involved, but a general formula is given by,

$$
\begin{aligned}
& \left\{G^{a, b}, G^{c, d}\right\}=a d G^{a-1, b} G^{c, d-1}-b c G^{a, b-1} G^{c-1, d} \\
& +\sum_{m=0}^{\left\lfloor\frac{M-1}{2}\right\rfloor}(-1)^{m} \frac{\hbar^{2 m}}{2^{2 m}} K_{a b c d}^{2 m+1} G^{a+c-2 m-1, b+d-2 m-1},
\end{aligned}
$$


where $M:=\operatorname{Min}(a+c, b+d, a+b, c+d)$ and the coefficients

$K_{a b c d}^{n}=\sum_{m=0}^{n}(-1)^{m} m !(n-m) !\left(\begin{array}{c}a \\ m\end{array}\right)\left(\begin{array}{c}b \\ n-m\end{array}\right)\left(\begin{array}{c}c \\ n-m\end{array}\right)\left(\begin{array}{c}d \\ m\end{array}\right)$

have been defined. Note that this expression mixes different orders in a nontrivial way since the bracket between two moments of order $(a+b)$ and $(c+d)$, respectively, are a combination of moments from at most order $|(a+b)-(c+d)|$ (or higher, depending which of the combinations in the definition of $M$ is the minimum), up to order $(a+b+c+d-2)$.

The evolution equations for different variables are then obtained by simply computing their Poisson brackets with the effective Hamiltonian (2). In this way, it is straightforward to get the following relations for the expectation values:

$$
\begin{aligned}
\frac{d q}{d t} & =\left\{q, H_{Q}\right\} \\
& =\frac{\partial H(q, p)}{\partial p}+\sum_{a+b \geq 2} \frac{1}{a ! b !} \frac{\partial^{a+b+1} H(q, p)}{\partial p^{a+1} \partial q^{b}} G^{a, b} \\
\frac{d p}{d t} & =\left\{p, H_{Q}\right\} \\
& =-\frac{\partial H(q, p)}{\partial q}-\sum_{a+b \geq 2} \frac{1}{a ! b !} \frac{\partial^{a+b+1} H(q, p)}{\partial p^{a} \partial q^{b+1}} G^{a, b}
\end{aligned}
$$

Note that if the classical Hamiltonian $H(q, p)$ is at most quadratic in the variables, the sums in these equations will give no contribution and the expectation values will exactly fulfill their corresponding classical equations of motion. In Sec. IIA this and other special features of quadratic Hamiltonians will be analyzed.

On the other hand, the equations of motion for the moments can be written as follows:

$$
\frac{d G^{a, b}}{d t}=\left\{G^{a, b}, H_{Q}\right\}=\sum_{c+d \geq 2} \frac{1}{c ! d !} \frac{\partial^{c+d} H}{\partial p^{c} \partial q^{d}}\left\{G^{a, b}, G^{c, d}\right\},
$$

where the Poisson bracket (4) should be replaced. As can be seen, in principle (except for certain particular forms of the Hamiltonian $H$ ) this equation will get contributions from several orders.

Even if the infinite set of equations is equivalent to the Schrödinger equation, for practical reasons in general, in order to analyze the dynamics of the system, it will be necessary to introduce a cut-off $>N$. That is, a maximum order $N$, so that all $G^{a, b}$ are assumed to be vanishing if $a+b>N$. Due to the special form of the brackets (4), that mix higher- and lower-order moments, the introduction of such a cut-off could be made in two different and inequivalent ways regarding the equations of motion for the moments (7). 1/ Truncate the Hamiltonian at order $N$, calculate the equations of motion (7), and truncate again the right-hand side of these equations. 2 / Consider the complete Hamiltonian $H_{Q}$ without truncation, calculate the equations of motion (7) and truncate then the result at order $N$. (In practice, for this second procedure it is enough to consider the Hamiltonian up to order $2 N$ since higher orders of the Hamiltonian will only introduce moments of an order greater than $N$ in the equations, which will be made to vanish when truncating the result.) On the other hand, the equations of motion for the expectation values $q$ and $p$ do not depend on the way the truncation is done. The truncation of the Hamiltonian at the desired order $N$ is sufficient to get only moments up to that order in their equations of motion and considering the full Hamiltonian $H_{Q}$ will not introduce new terms.

In principle, from a perturbative perspective, one would say that the first route is more consistent: no knowledge of higher-order terms is made use of at any step. Nevertheless, this is a very peculiar perturbative expansion since, as commented above, the Poisson brackets of a moment of order $N$ with a moment of order $N+1$ does not generate only moments of order $N$ and greater; lower-order terms also appear. Following the second route guarantees that the equations of motion truncated at a given order $N$ will contain all contributions from moments up to that order. That is, when considering the truncation at order $N+1$, the lower-order terms will not change.

This formalism is very practical because one deals directly with measurable quantities (expectation values) instead of with the wave function and it is immediate to see the corrections for classical equations of motion. These corrections are usually regarded as purely quantum but note that there is no explicit $\hbar$ involved in the definition of the moments or the expansion of the Hamiltonian. Only the Poisson brackets between two moments introduces $\hbar$ terms. A question then arises: is it possible to obtain a similar formalism for classical mechanics? As will be explained in the next section, the answer is in the affirmative.

\section{B. Classical moments}

As explained in [10], it is possible to define the classical analog of the moments define in the previous subsection. Let us assume a classical mechanical system described by the Hamiltonian $H$ with a phase space coordinatized by the conjugate pair $\tilde{q}$ and $\tilde{p}$ with Poisson bracket $\{\tilde{q}, \tilde{p}\}_{c}=$ 1 (the subscript $c$ is used not to confuse this bracket with the one defined previously for expectation values). A classical ensemble is defined via a probability distribution $\rho(\tilde{q}, \tilde{p}, t)$ on the classical phase space. The evolution of this distribution is given by the Liouville equation,

$$
\frac{\partial \rho}{\partial t}=-\{\rho, H(\tilde{q}, \tilde{p})\}_{c},
$$

which asserts that the probability distribution is conserved through physical trajectories $d \rho / d t=0$.

In order to explode further the parallelism between the classical and quantum worlds, the classical expectation 
value for any function in the phase space $f(\tilde{q}, \tilde{p})$ can be defined as

$$
\langle f(\tilde{q}, \tilde{p})\rangle_{\mathrm{c}}:=\int d \tilde{q} d \tilde{p} f(\tilde{q}, \tilde{p}) \rho(\tilde{q}, \tilde{p}, t),
$$

where the integration extends to the whole domain of the probability distribution on the phase space.

Making use of this operation, the classical mean values for the position $\tilde{q}$ and momentum $\tilde{p}$ are defined:

$$
\begin{aligned}
& q(t):=\langle\tilde{q}\rangle_{\mathrm{c}}, \\
& p(t):=\langle\tilde{p}\rangle_{\mathrm{c}} .
\end{aligned}
$$

These are the coordinates on the phase space of the centroid of our distribution. The classical moments are further defined as

$$
C^{a, b}:=\left\langle(\tilde{p}-p)^{a}(\tilde{q}-q)^{b}\right\rangle_{c} .
$$

Since all objects in this expression commute, contrary to the quantum case, the ordering is absolutely irrelevant. Note that the expectation value of any function can be regarded as a function of the coordinates of the centroid $(\tilde{q}, \tilde{p})$ and the moments $C^{a, b}$. To make this explicit it is enough to make a Taylor expansion around the position of the centroid:

$$
\begin{aligned}
\langle f(\tilde{q}, \tilde{p})\rangle_{\mathrm{c}} & =\langle f(q+\tilde{q}-q, p+\tilde{p}-p)\rangle_{\mathrm{c}} \\
& =f(q, p)+\sum_{a+b \geq 2} \frac{1}{a ! b !} \frac{\partial^{a+b} f(q, p)}{\partial q^{a} \partial p^{b}} C^{a, b}
\end{aligned}
$$

In order to obtain the equations of motion for different variables, one should define the following Poisson bracket between expectation values,

$$
\left\{\langle f(\tilde{q}, \tilde{p})\rangle_{c},\langle g(\tilde{q}, \tilde{p})\rangle_{c}\right\}:=\left\langle\{f(\tilde{q}, \tilde{p}), g(\tilde{q}, \tilde{p})\}_{c}\right\rangle_{c} .
$$

This operation has the good properties one expects for a bracket and gives the expected results for the expectation values of fundamental variables, now defined as the coordinates of the centroid of the distribution:

$$
\begin{array}{r}
\{q, p\}=\left\langle\{\tilde{q}, \tilde{p}\}_{c}\right\rangle_{c}=1, \\
\{q, q\}=0=\{p, p\} .
\end{array}
$$

As in the quantum case, the classical moments commute with these mean values,

$$
\left\{C^{a, b}, q\right\}=0=\left\{C^{a, b}, p\right\},
$$

and the Poisson bracket between any two classical moments is given as follows:

$$
\begin{aligned}
\left\{C^{a, b}, C^{c, d}\right\} & =a d C^{a-1, b} C^{c, d-1}-b c C^{a, b-1} C^{c-1, d} \\
& +(b c-a d) C^{a+c-1, b+d-1}
\end{aligned}
$$

Comparing these equations with the corresponding to the quantum moments (4), it is possible to see that the key difference between classical and quantum moments lies in the non-commutativity of the quantum operators. The first two summands of Eqs. (41) and (16) are identical, but the last term differs. In the quantum case there is a sum in even powers of $\hbar$ whereas in the classical case only the first summand, which is independent of $\hbar$, is kept. In the quantum case, these terms appear due to the non-commutativity of the basic operators and can be different for different orderings chosen in the definition of the moments. Nevertheless, it is not possible to make these terms vanishing by a redefinition of the ordering [10]. As a side remark, note that the Poisson bracket between a second-order moment and any other moment give the same result in the classical or in the quantum case. As it will be explained below, this fact will make the quantum evolution of the system up to second order completely equivalent to the classical evolution.

With these definitions at hand, the evolution of any expectation value $\langle f\rangle_{c}$ can be obtained in the following way,

$$
\begin{array}{r}
\frac{d\langle f\rangle_{c}}{d t}=\frac{d}{d t} \int d \tilde{q} d \tilde{p} \rho(\tilde{q}, \tilde{p}, t) f(\tilde{q}, \tilde{p}) \\
=-\int d \tilde{q} d \tilde{p}\{\rho(\tilde{q}, \tilde{p}, t), H(\tilde{q}, \tilde{p})\}_{c} f(\tilde{q}, \tilde{p}),
\end{array}
$$

where use of the Liouville equation has been made. Writing the Poisson bracket as derivatives with respect to the fundamental variables $(\tilde{q}, \tilde{p})$ and integrating by parts, it is easy to obtain

$$
\frac{d\langle f\rangle_{c}}{d t}=\int d \tilde{q} d \tilde{p} \rho\{f, H\}_{c}=\left\langle\{f, H\}_{c}\right\rangle_{c}=\left\{\langle f\rangle_{c},\langle H\rangle_{c}\right\} .
$$

This shows explicitly that the evolution of the classical ensemble described by the distribution $\rho(\tilde{q}, \tilde{p}, t)$ will be given by the expectation value of the classical Hamiltonian, which can be Taylor expanded around the centroid of the distribution:

$$
\begin{aligned}
H_{\mathrm{C}}\left(q, p, C^{a, b}\right) & :=\langle H(\tilde{q}, \tilde{p})\rangle_{\mathrm{c}} \\
& =\langle H(q+\tilde{q}-q, p+\tilde{p}-p)\rangle_{\mathrm{c}} \\
& =H(q, p)+\sum_{a+b \geq 2} \frac{1}{a ! b !} \frac{\partial^{a+b} H(q, p)}{\partial p^{a} \partial q^{b}} C^{a, b} .
\end{aligned}
$$

In particular, the equations of motion of the centroid are given by

$$
\begin{aligned}
\frac{d q}{d t} & =\left\{q, H_{\text {class }}\right\} \\
& =\frac{\partial H(q, p)}{\partial p}+\sum_{a+b \geq 2} \frac{1}{a ! b !} \frac{\partial^{a+b+1} H(q, p)}{\partial p^{a+1} \partial q^{b}} C^{a, b}, \\
\frac{d p}{d t} & =\left\{p, H_{\text {class }}\right\} \\
& =-\frac{\partial H(q, p)}{\partial q}-\sum_{a+b \geq 2} \frac{1}{a ! b !} \frac{\partial^{a+b+1} H(q, p)}{\partial p^{a} \partial q^{b+1}} C^{a, b} .
\end{aligned}
$$

It is clear from here that the centroid of the distribution does not generically follow a classical trajectory. Although in the particular case that one chooses a Dirac 
delta centered at $(\tilde{q}(0), \tilde{p}(0))$ as the initial distribution $\rho(\tilde{q}, \tilde{p}, t=0)$ all moments will vanish and this point would follow a classical trajectory. The first terms of the righthand side of both equations are the ones that corresponds to the usual Hamilton equations, but then there are corrections due to the moments. As in the quantum case, the moments and the mean values of the distribution form an infinite system of couple differential equations. The evolution of the classical moments is given by

$$
\frac{d C^{a, b}}{d t}=\sum_{c+d \geq 2} \frac{\partial^{c+d} H}{\partial p^{c} \partial q^{d}}\left\{C^{a, b}, C^{c, d}\right\} .
$$

These equations of motion can be recovered from the evolution equations of the quantum moments (7) simply by imposing $\hbar=0$.

\section{Classical versus quantum dynamics}

Note that remarkably, due to the properties of the Poisson brackets between quantum expectation values, there is no $\hbar$ term in the equations for second-order quantum moments (7) nor in the equations for the expectation values $q$ and $p$ (5) 6). Therefore, a truncation at second order give the same set of equations both for quantum and classical variables and thus it is necessary to study at least a third-order truncation in order to find differences between the classical and quantum evolutions.

The evolution equations for moments of higher order will in general be different due to the explicit $\hbar$ terms that appear in the equations for quantum moments (7). In addition, all equations are coupled and when considering a third (or higher) order truncation the evolution of expectation values $(q, p)$ and of second-order moments will be affected due to the back-reaction of other variables. In this case their evolution with the classical or quantum system will differ even if their equations of motion still have the same formal form.

One important conclusion of this analysis is that quantum effects have two different origins. On the one hand, there are distributional effects due to the fact that in the quantum theory one necessarily deals with the evolution of a spread probability distribution, as opposed to a point trajectory, on the phase space and thus moments are non-vanishing. As has been explained, these effects are also present in the classical setting when considering the evolution of a probability distribution on the phase space as correction terms to the usual Hamilton equations. On the other hand, the non-commutativity (or purely-quantum) effects appear in the quantum equations of motion through explicit $\hbar$ terms. Their origin is the non-commutativity of basic operators $\hat{q}$ and $\hat{p}$, and they are not present in the classical setting. On the contrary to the distributional effects, that may appear for instance when the initial conditions of a classical system are known only with certain finite precision, these latter effects are thus genuinely quantum.
It is difficult to measure the relative strength and importance of each effect since all equations are coupled and therefore both effects mix together. Intuitively the distributional effects depend on the peakedness of the distribution since, in general, the more spread the distribution the larger the magnitude of different moments. The noncommutativity effects enter in the equations of motion as a power series in $\hbar^{2}$, so the value of Planck constant can give an estimate of their relative importance.

An idea that will be pursued in a forthcoming article [20] is to analyze three different evolutions of the system: the classical and quantum evolution of the same initial distribution, as well as the classical point trajectory (taking all moments to vanish). The comparison between the classical point trajectory and the classical evolution of the expectation values with non-vanishing moments will estimate the strength of distributional effects, whereas the comparison between the quantum and classical evolutions will estimate the magnitude of the non-commutativity effects.

\section{Stationary and dynamical states}

Once the equations of motion for the Hamiltonian system have been obtained [Eqs. (55) f] for the quantum setting and (21] 23) for the classical one], the complete evolution of a dynamical state can be computed by solving them. The only additional physical input that is needed are the initial conditions for both the expectation values and the moments.

Nevertheless, the stationary states of a quantum system are also of remarkable importance. These states are solutions of the time-independent Schrödinger equation and, hence, eigenstates of the Hamiltonian operator. Their unique time dependence is encoded on a pure-phase term $e^{-i E t / \hbar}$. In the present formalism, based on a decomposition in moments of the wave function, it is possible to obtain the stationary states by analyzing the dynamical system given by the infinite set of moments (or, approximately, by its truncated version). In fact, there is no need to solve any differential equation: it is enough to equal to zero the right-hand side of all equations of motion (5,77) and solve the algebraic system in order to obtain the critical or equilibrium points. [The same analysis can be applied to Eqs. (21,23) to obtain the stationary classical distributions.] These equilibrium points are the equivalent of the quantum stationary states. In general, these equilibrium points would differ from the ones obtained in the case of a Dirac delta distribution with vanishing moments $C^{a, b}=0=G^{a, b}$, since the equations of motion of both $q$ and $p$ get corrections by moments.

However, in some cases the commented algebraic system of equations will not be complete [20], in such a way that will not be possible to find all moments, and additional relations must be found. Since this stationary states are eigenstates of the Hamiltonian operator, they obey that $\langle\hat{H}\rangle=E$, and in fact it is possible to find easily 
additional restrictions for them. For instance, the expectation value $\langle[\hat{H},[\hat{H}, g(\hat{q})]]\rangle$ must vanish for such a state. For the particular case of the Hamiltonian of a particle in a potential, $\hat{H}=\hat{p}^{2} / 2+V(\hat{q})$, that condition can be rewritten in the following way [21 23]:

$$
2 E\left\langle g^{\prime \prime}\right\rangle-2\left\langle g^{\prime \prime} V\right\rangle-\left\langle g^{\prime} V^{\prime}\right\rangle+\frac{\hbar^{2}}{4}\left\langle g^{\prime \prime \prime \prime}\right\rangle=0,
$$

for any $g(\hat{q})$. If the potential is polynomial in $\hat{q}$, for instance $V(\hat{q})=\hat{q}^{m}$, the free function can be chosen as $g(\hat{q})=(\hat{q}-q)^{n+2} /(n+2)$ and, taking into account the relation

$$
\left\langle\hat{q}^{n}\right\rangle=\sum_{m=0}^{n} q^{m} G^{0, n-m},
$$

write down a recursive relation for moments of the form $G^{0, n}$. In the particular case that the expectation value is vanishing $q=0$, the recursive relation takes the following form:

$$
\begin{aligned}
(2 n+m+2) G^{0, n+m} & =2 E(n+1) G^{0, n}+ \\
& +\frac{\hbar^{2}}{4}(n+1) n(n-1) G^{0, n-2} .
\end{aligned}
$$

This means that if moments up to order $G^{0, m}$ are known, the higher-order fluctuations can be obtained through this recursive relation. The equivalent computation for the classical moments gives the same recursive relation but with a vanishing $\hbar=0$. Therefore, the classical moments will obey a simpler (two-point) recursive relation.

Finally, other states of interest are the coherent states, which are defined as quantum dynamical states with an almost classical behavior. More precisely, their expectation value follow a classical trajectory on the phase space, whereas all their quantum moments are constant. Usually the value of the moments is taken to be of minimal uncertainty. This kind of states can only be constructed for the harmonic oscillator exactly. Although it might be possible to introduce a generalization of these states allowing for a relaxation of some of the commented features.

\section{SPECIAL HAMILTONIANS}

In this section two different class of Hamiltonians will be analyzed that have a very particular properties regarding the classical and quantum evolution they generate. On the one hand, as will be explained below, harmonic Hamiltonians (that are at most quadratic in both basic variables $q$ and $p$ ) essentially generate the same classical and quantum dynamics [24-26]. On the other hand, Hamiltonians that are linear in one of the basic variables induce exactly the same classical and quantum evolution on the expectation values $q$ and $p$, as well as on an infinite set of moments.

\section{A. Harmonic Hamiltonians}

Let us assume that we have a quantum Hamiltonian $H(q, p)$ that is at most quadratic in both basic variables $q$ and $p$. The effective Hamiltonian will then be given by

$H_{Q}=H(q, p)+\frac{1}{2} \frac{\partial^{2} H}{\partial p^{2}} G^{2,0}+\frac{\partial^{2} H}{\partial q \partial p} G^{1,1}+\frac{1}{2} \frac{\partial^{2} H}{\partial q^{2}} G^{0,2}$.

In this particular case the equations of motion both for the classical and quantum expectation values take the following form:

$$
\begin{gathered}
\frac{d q}{d t}=\frac{\partial H(q, p)}{\partial p} \\
\frac{d p}{d t}=-\frac{\partial H(q, p)}{\partial q} .
\end{gathered}
$$

These are the classical equations of motion for a point on the phase space, and do not get any correction term from the moments. In addition, the evolution equation for the moments can be written as

$$
\begin{aligned}
\frac{d G^{a, b}}{d t} & =b \frac{\partial^{2} H}{\partial p^{2}} G^{a+1, b-1}+(b-a) \frac{\partial^{2} H}{\partial p \partial q} G^{a, b} \\
& -a \frac{\partial^{2} H}{\partial q^{2}} G^{a-1, b+1} .
\end{aligned}
$$

It is easy to check that the equations of motion corresponding to the moments of a classical ensemble have the same structure as those for quantum variables just by replacing $G^{a, b}$ by $C^{a, b}$ in the last relation.

Therefore the dynamics generated by the harmonic Hamiltonian obey several special properties:

- On the one hand, the system of equations turns out to be linear (both in moments as well as in $q$ and $p$ variables) and with constant coefficients. Hence, the system can be easily solved analytically.

- On the other hand, all orders decouple: the equation of motion for a moment of $\mathcal{O}(a+b)$ only contains moments of that order.

- In particular, as we have already commented, there appears no moment on the Eqs. (27) and (28) so there is no back-reaction of the moments on the trajectories followed by the mean values. Therefore, the centroid of the distribution will exactly follow a classical dynamical (point) trajectory in phase space.

- In the general case, only the full $H_{Q}$ (and for the classical treatment $H_{C}$ ) is a constant of motion. But in this particular case, due to the decoupling of the equations of the centroid with the moments, $E_{\text {centroid }}=H(q, p)$ is also a constant of motion. This equality indeed describes the classical dynamical trajectory. Therefore, in addition, this leads to another conserved quantity: the combination of moments given by $H_{Q}-H(q, p)$ (and $H_{C}-H(q, p)$ for the classical moments). 
- In this case all equations are independent of $\hbar$. Therefore the evolution equations for the quantum and classical moments coincide. Hence, given the same initial data for classical and quantum probability distributions, the value of the moments and the coordinates of the centroid will coincide for all times.

- Finally, as will be made explicit in Subsec. IVE the combination of moments that appear in the Heisenberg inequality is conserved through evolution: $\frac{d}{d t}\left[G^{2,0} G^{0,2}-\left(G^{1,1}\right)^{2}\right]=0$. Therefore, if we choose a state that saturates the uncertainty relation as initial state, it will be kept saturated during the whole evolution.

All these features explain, in this context, the possibility of constructing exact dynamical coherent states for Hamiltonians of this form, whose centroid follows exactly a classical (point) trajectory in phase space [27].

Nevertheless, a quantum harmonic oscillator is very different from a classical oscillator. Even if, given the same initial data, the dynamical (distributional) states are similar due to all the properties explained above, the moments corresponding to stationary states differ as an even-power series in $\hbar$ due to the recursive relation (26). It is straightforward to see that it is necessary to make use of this recursive relation since the algebraic equations that are obtained by equaling to zero the right-hand side of Eq. (29) are not linearly independent [20].

\section{B. Linear Hamiltonians}

Another set of Hamiltonians that turn out to be very interesting due to the properties of the equations it generates is given by those Hamiltonians that are linear in one of the basic variables. For definiteness, let us assume a Hamiltonian that is linear in the position $q$ but have a general dependence on the moment $p$. Its most general form is given by,

$$
H=q \varphi(p)+\xi(p)
$$

for arbitrary functions $\varphi$ and $\xi$. Note that the case given by a linear function $\varphi$ and a quadratic function $\xi$ is included in the previous subsection about harmonic Hamiltonians. The generalization for a Hamiltonian linear in $p$ is straightforward.

The effective quantum Hamiltonian is given by,

$$
\begin{aligned}
H_{Q} & =q \varphi(p)+\xi(p) \\
& +\sum_{n=1}^{\infty} \frac{1}{(n+1) !}\left[q \varphi^{(n+1)}(p)+\xi^{(n+1)}(p)\right] G^{n+1,0} \\
& +\frac{\varphi^{(n)}(p)}{n !} G^{n, 1}
\end{aligned}
$$

From here it is straightforward to obtain the equations of motion for expectation values:

$$
\begin{aligned}
\frac{d q}{d t} & =q \varphi^{\prime}(p)+\xi^{\prime}(p) \\
& +\sum_{n=1}^{\infty} \frac{1}{(n+1) !}\left[q \varphi^{(n+2)}(p)+\xi^{(n+2)}(p)\right] G^{n+1,0} \\
& +\frac{\varphi^{(n+1)}(p)}{n !} G^{n, 1} \\
\frac{d p}{d t} & =-\varphi(p)-\sum_{n=2}^{\infty} \frac{1}{n !} \varphi^{(n)}(p) G^{n, 0}
\end{aligned}
$$

Contrary to the harmonic Hamiltonians, in this case the equations of motion for expectation values do get contributions from certain moments and, therefore, the centroid of the system will not generically follow a classical orbit on the phase space. In spite of this, the system under consideration have very peculiar properties. Let us compute the equations of motion for the moments that appear in the equations above, namely $G^{a, 0}$ and $G^{b, 1}$ :

$$
\begin{aligned}
\frac{d G^{a, 0}}{d t} & =a \sum_{n=1}^{\infty} \frac{\varphi^{(n)}(p)}{n !}\left[G^{a-1,0} G^{n, 0}-G^{a+n-1,0}\right], \\
\frac{d G^{b, 1}}{d t} & =\sum_{n=1}^{\infty} \frac{1}{n !}\left[q \varphi^{(n+1)}(p)+\xi^{(n+1)}(p)\right]\left(G^{b+n, 0}-G^{b, 0} G^{n, 0}\right) \\
& +\frac{\varphi^{(n)}(p)}{n !}\left[b G^{b-1,1} G^{n, 0}-n G^{b, 0} G^{n-1,1}+(n-b) G^{b+n-1,1}\right]
\end{aligned}
$$

In obtaining these equations it is useful to note that Poisson brackets between moments $G^{a, 0}$ and $G^{b, 0}$ is vanishing for any value of $a$ and $b$. For generic functions $\varphi$ and $\xi$, these equations are quite involved. Nevertheless, the equations for the expectation values 32 , 33 , in combination with those for moments of the form $G^{a, 0}$ and $G^{b, 1}$ (34) 35) constitute a closed, though infinite, system of differential equations. Note that even if the derivatives of 
the functions $\varphi^{(n)}$ and $\xi^{(n)}$ are vanishing for all $n$ greater or equal to certain value $n_{\max }$ the mentioned subsystem will only close by considering infinite moments. Therefore there is no decoupled finite system. Let us explain this in more detail. If $\varphi^{(n)}=0$ for all $n>n_{\max }$ only moments $G^{a, 0}$ and $G^{b, 1}$ up to order $n_{\max }$ would appear in the equations for the expectation values. But, for instance, in the equation for $G^{n_{\max }, 0}$ higher-order moments will appear due to the last term in Eq. (34) and thus the finite system will not close.

In any case, this is an interesting observation since one does not need to consider all infinite moments in order to obtain the trajectory of the centroid of the distribution. In fact a decoupled system is formed for expectation values $(q, p)$, and moments $\left(G^{a, 0}, G^{a, 1}, G^{a, 2}, \ldots, G^{a, m}\right)$ for all $a$ and a fixed $m \geq 1$. This gives a way to solve the infinite system. One can first solve for the system given by $\left(q, p, G^{a, 0}, G^{a, 1}\right)$. Once this is known, it is possible to solve for all $G^{a, 2}$ making use of the previous solution, and so on. Even though, in practice this method is hardly applicable since all those are, in principle, infinite systems.

In addition to the commented features, there is no $\hbar$ term present in any of the Eqs. (32 35). Therefore, the moments $C^{a, 0}$ and $C^{b, 1}$ as well as the expectation values $q$ and $p$ corresponding to the classical distribution will follow the same equations as their quantum counterparts. This means that for Hamiltonians of the form (31), the centroid of the quantum distribution will not follow a classical orbit in the phase space but will indeed follow the trajectory given by the centroid of the classical distribution with same initial conditions. Thus, in these quantum systems the departure from a classical orbit on phase space is completely due to distributional effects that are also present in the evolution of a classical distribution.

Nevertheless, the evolution of moments that are not of the form $G^{a, 0}$ or $G^{a, 1}$ will be different for the classical and quantum distributions due to the $\hbar$ terms that appear in the quantum equations of motion. Therefore, in order to measure pure-quantum effects it is necessary to consider the evolution of such moments. [Note that, even if in the particular case of the equation of motion for $G^{0,2}$ there is no explicit $\hbar$ term, its evolution differs from its background counterpart $C^{0,2}$ because it is coupled with other moments that indeed present pure-quantum terms in their equations of motion.]

Technically, the form of these equations and particularly the decoupling of equations for the variables $\left(q, p, G^{a, 0}, G^{b, 1}\right)$, for all $a$ and $b$, from the rest of the moments can be traced back to the fact that the mentioned moments form a closed Poisson algebra:

$$
\begin{aligned}
\left\{G^{a, 0}, G^{b, 0}\right\} & =0 \\
\left\{G^{a, 0}, G^{b, 1}\right\} & =a\left(G^{a-1,0} G^{b, 0}-G^{a+b-1,0}\right), \\
\left\{G^{a, 1}, G^{b, 1}\right\} & =a G^{a-1,1} G^{b, 0}-b G^{a, 0} G^{b-1,1} \\
& +(b-a) G^{a+b-1,1} .
\end{aligned}
$$

This does not happen if one considers also moments with a higher index in the position variable. Already including moments of the form $G^{a, 2}$ would make the Poisson algebra not to close. It is easy to see from the general form of the brackets (4) that even if the Poisson brackets between moments of the form $G^{a, 0}$ and $G^{a, 1}$ with those of the form $G^{b, 2}$ do close, the brackets between two moments of the form $G^{a, 2}$ give other kind of contributions. For instance:

$$
\left\{G^{1,2}, G^{0,2}\right\}=-2 G^{0,3} .
$$

Therefore, instead of being linear if the Hamiltonian was quadratic in the position $q$, moments of the form $G^{a, 2}$ would appear in the effective Hamiltonian. And this fact would not permit a decoupling of the equations of motion similar to the one explained above.

Finally, let us briefly explain the particular case of a Hamiltonian that depends only on one of the two basic variables. Following with the notation above, this corresponds to $\varphi(p)=0$. In this case, the procedure explained above is very useful. The momentum $p$ and all its pure fluctuations $G^{a, 0}$ turn out to be constants of motion. From Eq. (35), it is easy to solve for moments $G^{a, 1}$, obtaining a linear dependence with time,

$$
G^{a, 1}(t)=G^{a, 1}\left(t_{0}\right)+\Phi\left(p, G^{a, 0}\right)\left(t-t_{0}\right),
$$

with a function $\Phi$ that depends on the constants of motion. With this solution at hand one can solve for $G^{a, 2}$, which will give a dependence quadratic in time. The procedure can be iterated for higher values of the index of the position and obtain that a generic moment $G^{a, b}$ will have a polynomial dependence on time of order $\left(t-t_{0}\right)^{b}$. Explicit $\hbar$ terms, that will encode the differences between the classical and quantum evolution, first appear for moments of the form $G^{a, 3}$.

\section{INEQUALITIES FOR STATISTICAL MOMENTS}

Any set of numbers $M^{a, b}$ does not need to correspond to statistical moments $G^{a, b}$ of a given probability distribution function. Indeed, they need to obey certain inequalities. In this section we will derive several set of inequalities starting from the Cauchy-Schwarz inequality. In particular, we will derive the generalized inequality relations for quantum moments, that do not allow all moments to be vanishing.

This analysis has theoretical importance by its own in order to know what kind of distributions are allowed. Furthermore, it will provide us with additional information that constraint the values of high-order moments. This information could be used for different purposes in many scenarios. For example to constraint the moments corresponding to a stationary state [20], in the construction of effective group coherent states [28], or to control the validity of a prospective numerical implementation of the system. 
In addition, they could also be used to generate physically viable random initial data in order to check the evolution of the complete parameter space. Nonetheless, as will be commented below, it is not completely guaranteed that if a set of numbers $M^{a, b}$ obey the inequalities that will be derived here, they will correspond to the moments of valid probability distribution.

By construction, we have that both for quantum and classical variables, the moments with both even indices must be positive definite:

$$
\begin{aligned}
& C^{2 n, 2 m} \geq 0, \\
& G^{2 n, 2 m} \geq 0, \text { for } n, m \in \mathbb{N} .
\end{aligned}
$$

\section{A. Inequalities for the classical moments}

In order to derive the rest of the inequalities, we will make use of the Cauchy-Schwarz inequality that, for the classical expectation value operation, takes the following form,

$$
\langle f g\rangle_{c}^{2} \leq\left\langle f^{2}\right\rangle_{c}\left\langle g^{2}\right\rangle_{c}
$$

$f$ and $g$ being any function of the position $q$ and momentum $p$. We will make two different choices for those functions: $i / f=(p-\langle p\rangle)^{a}(q-\langle q\rangle)^{b}$ and $g=$ $(p-\langle p\rangle)^{c}(q-\langle q\rangle)^{d}$, and ii/ $f=[(q-\langle q\rangle)+(p-\langle p\rangle)]^{a}$ and $g=[(q-\langle q\rangle)+(p-\langle p\rangle)]^{b}$. These forms of functions are chosen because, as will be shown below, they lead to a sufficiently large class of inequalities that heavily constraint the values of moments at different orders and give us some information about the kind of distributions that are allowed. Nevertheless, Cauchy-Schwarz inequality is obeyed for any pair of functions, $f$ and $g$, and thus we do not have the certainty that we are saturating the complete information contained in that inequality by choosing the functions of the forms mentioned above. That is, in principle choosing moments that obey the inequalities we will derive will not completely guarantee that they correspond to a valid probability distribution.

The first choice, $f=(p-\langle p\rangle)^{a}(q-\langle q\rangle)^{b}$ and $g=$ $(p-\langle p\rangle)^{c}(q-\langle q\rangle)^{d}$, leads to the following relation:

$$
\left(C^{a+c, b+d}\right)^{2} \leq C^{2 a, 2 b} C^{2 c, 2 d},
$$

for all non-negative integers $a, b, c, d$. This relation can also be rewritten as,

$$
\left(C^{n, m}\right)^{2} \leq C^{2(n-c), 2(m-d)} C^{2 c, 2 d},
$$

for all $n \leq c$ and $m \leq d$. This is an infinite set of inequalities that, for its lowest order, reproduces the well-known relation between the covariance $C^{1,1}:=\langle(q-\langle q\rangle)(p-\langle p\rangle)\rangle$ between two random variables, in this case $q$ and $p$, and their corresponding variances $C^{0,2}=\langle(q-\langle q\rangle)$ and $C^{2,0}=\langle(p-\langle p\rangle)$ :

$$
\left(C^{1,1}\right)^{2} \leq C^{2,0} C^{0,2} .
$$

For quantum moments this particular relation, as will be explained below, will take the form of the Heisenberg uncertainty relation.

Note that even if we choose the indices of the moment that appear on the left-hand side of inequality (44), we are only imposing a fix value for the sums $a+c=n$ and $b+d=m$. Once this is fixed, the right-hand side can take $\lceil(n+1)(m+1) / 2\rceil$ different forms. Therefore, from Eq. (44) we obtain, for each $C^{n, m},\lceil(n+1)(m+1) / 2\rceil$ different quadratic combinations of moments that must be greater or equal to its square. For instance, let us choose the moment $a+c=1$ and $b+d=2$. This particular choice provides us with the following three independent relations:

$$
\begin{array}{r}
\left(C^{1,2}\right)^{2} \leq C^{2,4}, \\
\left(C^{1,2}\right)^{2} \leq C^{0,2} C^{2,2}, \\
\left(C^{1,2}\right)^{2} \leq C^{0,4} C^{2,0},
\end{array}
$$

where we have made use of the fact that $C^{0,0}=1$.

The second choice, $f=[(q-\langle q\rangle)+(p-\langle p\rangle)]^{a}$ and $g=[(q-\langle q\rangle)+(p-\langle p\rangle)]^{b}$, gives rise to the general inequality,

$$
\begin{aligned}
& {\left[\begin{array}{c}
a+b \\
\left.\sum_{n=0}\left(\begin{array}{c}
a+b \\
n
\end{array}\right) C^{n, a+b-n}\right]^{2}
\end{array}\right.} \\
& \leq \sum_{j=0}^{2 a} \sum_{k=0}^{2 b}\left(\begin{array}{c}
2 a \\
j
\end{array}\right)\left(\begin{array}{c}
2 b \\
k
\end{array}\right) C^{j, 2 a-j} C^{k, 2 b-k} .
\end{aligned}
$$

This inequality relates the square of a given sum that contains all moments of order $\mathcal{O}(a+b)$ to quadratic combinations of moments of order $\mathcal{O}(2 a)$ and $\mathcal{O}(2 b)$. Note that for the case $a=b$, both sides give the same result and hence this relation trivially reduces to an equality. In general, this inequality is independent of the previous one (44), but in some simple cases, it is contained in there. For instance, imposing $a=1$ and $b=0$, the left-hand side vanishes and we obtain

$$
0 \leq C^{0,2}+2 C^{1,1}+C^{2,0},
$$

which is trivially obeyed if these objets fulfilled relation (46).

In summary, the most important results of this section are the two infinite collections of inequalities, (44) and (48), that must be fulfilled by the classical statistical moments. These relations are direct consequence of the well-known Cauchy-Schwarz inequality but, up to the best of our knowledge, this is the first time in the literature that they are presented in this form.

\section{B. Inequalities for quantum moments}

Previous analysis should also be reproduced for quantum moments. In particular, we should obtain relations so that when $\hbar$ is taken to be vanishing, they reduce to 
the ones obtained in the previous subsection. As we will see, the $\hbar$ terms present in these quantum inequalities come from applying the Weyl symmetrization to reproduce the definition of the moments.

In this case Cauchy-Schwarz inequality is written as

$$
\left|\left\langle\hat{f}^{\dagger} \hat{g}\right\rangle\right|^{2} \leq\left\langle\hat{f}^{\dagger} \hat{f}\right\rangle\left\langle\hat{g}^{\dagger} \hat{g}\right\rangle
$$

where $\hat{f}$ and $\hat{g}$ are combinations of $\hat{q}$ and $\hat{p}$ operators. In order to generalize the inequalities derived for the classical moments, we will consider again the two different forms of operators $\hat{f}$ and $\hat{g}$ : on the one hand $i /[(\hat{q}-\langle\hat{q}\rangle)+(\hat{p}-\langle\hat{p}\rangle)]^{a}$ and, on the other hand, $i i /$ $(\hat{p}-p)^{a}(\hat{q}-q)^{b}$.

Interestingly, the first choice for the operators, $\hat{f}=$ $[(\hat{q}-\langle\hat{q}\rangle)+(\hat{p}-\langle\hat{p}\rangle)]^{a}$ and $\hat{g}=[(\hat{q}-\langle\hat{q}\rangle)+(\hat{p}-\langle\hat{p}\rangle)]^{b}$, leads formally to the same inequalities as their classical counterparts:

$$
\begin{aligned}
& {\left[\sum_{n=0}^{a+b}\left(\begin{array}{c}
a+b \\
n
\end{array}\right) G^{n, a+b-n}\right]^{2} \leq} \\
& \leq \sum_{j=0}^{2 a} \sum_{k=0}^{2 b}\left(\begin{array}{c}
2 a \\
j
\end{array}\right)\left(\begin{array}{c}
2 b \\
k
\end{array}\right) G^{j, 2 a-j} G^{k, 2 b-k},(51)
\end{aligned}
$$

which provides independent inequalities for all $b>a$. Note that in these relations there is no presence of $\hbar$. This is due to the fact that, for the above mentioned form of the operators, $\hat{f}$ and $\hat{g}$ are self-adjoint and their products $\hat{f} \hat{g}, \hat{f} \hat{f}$ and $\hat{g} \hat{g}$ are already in completely symmetrical Weyl ordering. Therefore, the definition of quantum moments is obtained without any use of the commutation relations.

On the contrary, the inequalities derived from the second choice of operators ii/ $\hat{f}=(\hat{p}-p)^{a}(\hat{q}-q)^{b}$ and $\hat{g}=(\hat{p}-p)^{c}(\hat{q}-q)^{d}$, for different values of the powers $(a, b, c, d)$ and with different orderings, will in general have explicit $\hbar$ terms.

Some particular cases that do not involve any $\hbar$ terms are those for which $\hat{f}$ and $\hat{g}$ commute, for instance $\hat{f}=$ $(\hat{p}-\langle\hat{p}\rangle)^{a}$ and $\hat{g}=(\hat{p}-\langle\hat{p}\rangle)^{d}$. In this way, we obtain the following general relations for quantum moments, that do not involve $\hbar$ and are formally the same as the classical inequalities:

$$
\begin{aligned}
\left(G^{a+b, 0}\right)^{2} & \leq G^{2 a, 0} G^{2 d, 0} \\
\left(G^{0, a+d}\right)^{2} & \leq G^{0,2 a} G^{0,2 b}
\end{aligned}
$$

The rest of the cases, that are not included in relations (52 53) are more complicated due to the noncommutativity of operators and involve new terms as a power series in $\hbar^{2}$. All of them can be formally written as

$$
\left(G^{a+c, b+d}\right)^{2} \leq G^{2 a, 2 b} G^{2 c, 2 d}+\sum_{n, i, j, k, l} \hbar^{2 n} \alpha_{a b c d}^{i j k l} G^{i, j} G^{k, l}{ }_{(54)}
$$

with certain coefficients $\alpha_{a b c d}^{i j k l}$. The sum on $n$ runs over all integers from 1 to the integer part of $(a+b+c+d) / 2$ and relation $4 n+i+j+k+l=2(a+b+c+d)$ is obeyed for all terms in the sum.

It is quite difficult to obtain the analytical form of the coefficients $\alpha_{a b c d}^{i j k l}$, thus we have obtained these relations by direct computation by making use of the iterative algebraic code presented in Ref. [15]. All combinations between operators of the form $\hat{f}=(\hat{p}-\langle\hat{p}\rangle)^{a}(\hat{q}-\langle\hat{q}\rangle)^{b}$ and $\hat{g}=(\hat{p}-\langle\hat{p}\rangle)^{a}(\hat{q}-\langle\hat{q}\rangle)^{b}$, for all internal orderings of operators $\hat{q}$ and $\hat{p}$, from first up to fifth order (that is from $a+b=1$ up to $a+b=5$, and the same for $c+d$ ) have been considered. In this way, we have obtained 1449 different inequalities that involve moments up to tenth order. All of them might not be independent and there are several inequalities that have the same classical limit, that is, with different coefficients $\alpha_{a b c d}^{i j k l}$. In those cases, one should take the most restrictive ones but this is not a simple analysis with so many variables. Therefore, for practical reasons, we keep all of them. In order to illustrate the form of these quantum inequalities here we will just show the quantum generalization of the particular examples shown in the previous subsection. In addition, in the next subsection, a particularly important set of inequalities will be analyzed: the uncertainty relations.

Regarding the examples shown explicitly in the previous subsection, on the one hand the classical relation between the covariance and the variance (46) is generalized by the Heisenberg uncertainty principle:

$$
\left(G^{1,1}\right)^{2} \leq G^{2,0} G^{0,2}-\frac{\hbar^{2}}{4} .
$$

On the other hand relations (47), derived from (44) for a left-hand side of the form $\left(C^{1,1}\right)^{2}$, are generalized by the following inequalities between quantum moments:

$$
\begin{aligned}
& \left(G^{1,2}\right)^{2} \leq G^{2,4}+\hbar^{2} G^{0,2} \\
& \left(G^{1,2}\right)^{2} \leq G^{0,2} G^{2,2}+\frac{\hbar^{2}}{2} G^{0,2} \\
& \left(G^{1,2}\right)^{2} \leq G^{0,4} G^{2,0}
\end{aligned}
$$

As can be seen, some inequalities, as the last one, do not change and keep the same form as the one corresponding to the classical moments.

\section{Uncertainty relations}

It is interesting to note that in the classical setting, a Dirac delta distribution is consistent with all derived inequalities. That is, it is possible to take all $C^{a, b}$ moments to be vanishing. On the contrary, as it is well known from the Heisenberg uncertainty principle, in the quantum case the limit $G^{a, b} \rightarrow 0$ is not consistent.

In fact, most of derived inequalities for quantum moments allow for a distribution given by Dirac delta; that is, they are not violated if one imposes all quantum moments to be vanishing. Heisenberg uncertainty relation (55) is the most simple inequality that does not allow for 
such a limit. In addition to that, among the 1449 different relations that have been obtained, there are 160 relations that forbids the mentioned limit. These relations are the ones that, as well as the uncertainty relation, encode the necessary lack of information of the quantum system an observer must have.

All uncertainty relations come from the quantum version of the classical inequalities (45) with $n=m$ and can be formally written as

$$
\begin{aligned}
\left(G^{n, n}\right)^{2}+\gamma_{n c d} \hbar^{2 n} & \leq G^{2(n-c), 2(n-d)} G^{2 c, 2 d}+ \\
& +\sum_{i=1}^{n-1} \alpha_{n i j k l m} \hbar^{2 i} G^{j, k} G^{l, m}
\end{aligned}
$$

Interestingly the uncertainty relations are only those inequalities for which $c \neq d$, since in that case the constant $\gamma_{n c d}$ turns out to be positive. The case $c=d=n / 2$, for even $n$, trivially reduces to an equality; whereas for the rest of the $c=d$ cases, $\gamma_{n c d}$ is a negative constant and thus does not constitute an uncertainty relation since it is obeyed when choosing all $G^{a, b}$ moments to be vanishing. Uncertainty relations with $n=1$ and $n=2$ are explicitly shown in the appendix.

Essentially, all these uncertainty relations bound from below products of the form $G^{2 a, 0} G^{0,2 a}$. In fact, it is possible to choose all moments vanishing except $G^{2 a, 0}$ and $G^{0,2 a}$. Under this particular choice, the 160 uncertainty relations reduce to five inequalities of the form,

$$
\gamma_{n} \hbar^{2 n} \leq G^{0,2 n} G^{2 n, 0}
$$

with a positive constant $\gamma_{n}$ for each value of $n$. More precisely: $\gamma_{1}=1 / 4, \gamma_{2}=3 / 8, \gamma_{3}=81 / 64, \gamma_{4}=9 / 4$, and $\gamma_{5}=225 / 16$.

Following with this choice of moments (all vanishing except $G^{2 a, 0}$ and $G^{0,2 a}$ ), one could choose to have the same uncertainty both in momentum and position by imposing $G^{2 a, 0}=G^{0,2 a}=g_{a} \hbar^{a}$ and uncertainty relations (57) will simply impose a minimum value for each adimensional moment $g_{n}$. In addition to those inequalities, it is also necessary to take into account the rest of the quantum inequalities that have been derived in the previous section. In particular, it is interesting to note that with this simple choice all quantum inequalities that contain up to eighth-order moments are reduced to the following constraints on the adimensional moments $g_{a}$ :

$$
g_{1} \geq \frac{1}{2}, \quad g_{2} \geq 6\left(g_{1}\right)^{2}, \quad g_{3} g_{1} \geq \frac{9}{4}\left(g_{2}\right)^{2}, \quad g_{4} g_{2} \geq \frac{25}{14}\left(g_{3}\right)^{2} \text {. }
$$

If one wishes to find a minimum value for the moments, as can be seen in this expression, one would have to face the tension that exist between lower-order moments and higher-order ones. For instance, from the third inequality with a fixed value of $g_{2}$, the lower $g_{1}$ the larger $g_{3}$ will have to be chosen. In any case, one could choose the saturation of every inequality starting from the lowest one, which is not affected by higher-order moments, and solve the inequalities order by order. Nevertheless, as has been commented on the previous subsection, there is no certainty that solving all derived inequalities will lead to a valid state since there could be other constraints on the moments that have not been considered.

\section{Other allowed distributions}

Apart from the vanishing moments limit of a Dirac delta, there are other distribution of moments that are allowed by the classical inequalities we have derived, but not by the quantum ones. This is the case for instance of $C^{a, b}=\hbar^{(a+b) / 2}$, or $C^{a, b}=A^{a+b} \hbar^{(a+b) / 2}$, with an adimensional constant $A$. (The factor $\hbar^{(a+b) / 2}$ stands for dimensional reasons.)

Apparently, in order to fulfill the corresponding inequalities, the quantum moments need a factor in front of $\hbar^{(a+b) / 2}$ that increases faster than $A^{a+b}$ with the indices $a$ and $b$. In particular, the following distributions are allowed both by classical and quantum inequalities:

$$
\begin{array}{r}
G^{a, b}=a ! b ! \hbar^{(a+b) / 2}, \\
G^{a, b}=a^{a} b^{b} \hbar^{(a+b) / 2}, \\
G^{a, b}=a^{a-1} b^{b-1} \hbar^{(a+b) / 2} .
\end{array}
$$

Interestingly the distribution $G^{a, b}=a^{a-2} b^{b-2} \hbar^{(a+b) / 2}$ is allowed by classical but not by quantum inequalities; whereas $G^{a, b}=a^{a-3} b^{b-3} \hbar^{(a+b) / 2}$ is neither allowed by quantum nor by classical relations.

On the other hand, defining the moments as functions of their order only, like $G^{a, b}=(a+b) ! \hbar^{(a+b) / 2}$ obey all inequalities except Heisenberg uncertainty relation.

Finally all these considerations leads to think that the quantum moments necessarily form a divergent series. If we assume that this divergent series is asymptotic, it is possible to analyze up to which order they converge, that is, which will the smallest term of the series. Usually the truncation at that order is the optimal truncation in the sense that the accuracy of the result improves as one includes more and more orders up to the mentioned smallest term. From that order on, adding more orders will worsen the result. For instance, for the distribution given by $a ! b ! \hbar^{(a+b) / 2}$, two moments of consecutive orders $G^{n-1,0}$ and $G^{n, 0}$ will be of the same order when $n=$ $1 / \sqrt{\hbar}$. Therefore, the smaller the value of $\hbar$ is chosen, the more orders can be considered in order to improve the result.

\section{E. Evolution of the uncertainty relations}

Let us analyze the evolution of the Heisenberg uncertainty principle. Given a generic Hamiltonian $H(q, p)$, it is straightforward to obtain 


$$
\frac{\mathrm{d}}{\mathrm{d} t}\left[G^{2,0} G^{0,2}-\left(G^{1,1}\right)^{2}\right]=\sum_{a+b \geq 3} \frac{2}{a ! b !} \frac{\partial^{a+b} H}{\partial p^{a} \partial q^{b}}\left[a G^{2,0} G^{a-1, b+1}-b G^{0,2} G^{a+1, b-1}+(b-a) G^{1,1} G^{a, b}\right]
$$

where the sum is for all non-negative $a$ and $b$ that obeys the constraint $a+b \geq 3$. It is interesting to note that in the right-hand side of that expression only derivatives of the Hamiltonian higher than two appear. In the evolution equation for each of the moments second-order derivatives of the Hamiltonian are present but, for that specific combination of moments, these terms cancel each other. Therefore, this leads to another important property of harmonic Hamiltonians: the combination of moments in the Heisenberg uncertainty principle is a constant of motion. Certainly, this does not mean that the initial state will not be deformed through evolution. All moments will generically grow in absolute value with time but keeping that relation constant. For squeezed states, defined as those that saturate Heisenberg uncertainty relation, this saturation will be kept throughout evolution. However, this property does not apply to the higher-order uncertainty relations that have been presented in the previous subsection. The combination of moments appearing in those relations are not generically conserved even for harmonic Hamiltonians.

On the other hand, as a general feature of secondorder moment evolution, there are no $\hbar$ terms present in Eq. (61). Thus for second-order moments the classical and quantum equations of motion are exactly the same, and the above considerations also apply to the relation $C^{0,2} C^{2,0}-\left(C^{1,1}\right)^{2} \geq 0$ between classical moments. This, of course, does not mean that considering the full dynamics under a generic non-harmonic Hamiltonian the behavior of the classical and quantum system will be the same. The quantum corrections enter at third order and, due to the back-reaction, they will also affect the evolution of background quantities and second-order moments.

\section{CONCLUSIONS}

Quantum effects have two different origins. On the one hand, distributional effects are a direct consequence of the Heisenberg uncertainty relation, since the state of a quantum system is necessarily given by an extended probability distribution, instead of a single point in phase space. Technically this means that the statistical moments that describe this distribution can not be vanishing, and they back-react on the evolution of the position of the centroid. Thus generically the centroid of a quantum distribution does not follow a classical (point) trajectory in the phase space. On the other hand, due to the non-commutativity of the basic quantum operators, the non-commutative effects appear in the evolution equations of quantum statistical moments as terms that come with an explicit dependence on $\hbar$. Due to the smallness of this constant, the distributional effects may be more important than these latter ones. In the formalism used in this paper, based on a decomposition on statistical moments of the wave function, the distinction between these two sources of quantum corrections is very neat.

Furthermore, the evolution of a classical probability distribution has also been considered in terms of its statistical moments. This is particularly important since, even if in the context of classical mechanics is ideally possible to study the evolution of an initial point in the phase space, in practice there are always errors in the initial conditions and thus one needs to deal with an extended probability distribution. In the classical case, the distributional effects are also present and generically the centroid of a distribution does not either follow a classical orbit in the phase space. In fact, the only difference between the quantum and classical evolution equations are the explicit $\hbar$ factors that appear in the quantum system. Therefore, when taking the $\hbar \longrightarrow 0$ limit on the quantum system, one does not recover a unique classical trajectory on the phase space, but an ensemble of them.

There are some Hamiltonians that have very peculiar properties regarding the classical and quantum evolution they generate. The harmonic Hamiltonians, those that are at most quadratic on the basic variables, are very special. One of their most important property is that they generate the same dynamics both in the classical and quantum sector. The Hamiltonians that are linear in one of the basic variables also generate the same classical and quantum flux for the position of the centroid of the distribution and certain infinite set of moments. Therefore, in order to look for pure-quantum effects in this kind of systems, one should check the evolution of moments that are not contained in that set.

Finally, a large set of inequalities obeyed by classical and quantum moments have been obtained by making use of the Cauchy-Schwarz inequality. Among these, the uncertainty relations obeyed by quantum moments, that are defined as those that do not allow for all moments to be vanishing. The simplest of these relations is the wellknown Heisenberg uncertainty principle. The rest constitute its higher-order generalizations. In essence they provide additional information that constraint the value of high-order moments. This information could be used in different analysis; for instance, to constraint the value of the moments corresponding to a stationary state 20], in the construction of effective group coherent states [28], or to control the validity of a prospective numerical implementation of the system. The distribution of moments allowed by these inequalities have also been analyzed, as 
well as the evolution of the uncertainty relations under generic Hamiltonians.

As future work, the formalism developed in this article will be applied to different simple physical systems, like the harmonic and quartic oscillators, in order to measure the strength of each kind of quantum effect and to obtain the statistical moments corresponding to their (classical and quantum) stationary states [20]. It would also be interesting to revisit the different cosmological models commented in the introduction, like the ones studied in [14 16], to compare their quantum and classical distributional evolution.

\section{Acknowledgments}

The author thanks Carlos Barceló, Raúl CarballoRubio, Iñaki Garay, Claus Kiefer, Manuel Krämer, and Hannes Schenck for discussions and comments. Special thanks to Martin Bojowald for interesting comments on a previous version of this manuscript. Financial support from the Alexander von Humboldt Foundation through a postdoctoral fellowship is gratefully acknowledged. This work is supported in part by Projects IT592-13 of the Basque Government and FIS2012-34379 of the Spanish Ministry of Economy and Competitiveness.

\section{Appendix A: Uncertainty relations}

In order not to extend this appendix in excess, here the uncertainty relations that contain moments only up to sixth order are presented. The generalized uncertainty relations, defined as those inequalities that are not obeyed for the case $G^{a, b}=0$, are the quantum equivalent of the inequalities (44) for classical moments and can be generically written as shown in (56). In particular, the well-known Heisenberg uncertainty relation is given as,

$$
\hbar^{2} / 4+\left(G^{1,1}\right)^{2} \leq G^{0,2} G^{2,0} .
$$

The generalizations of $\left(C^{2,2}\right)^{2} \leq C^{2 a, 2 c} C^{2 b, 2 d}$ with $a+$ $b=2$ and $c+d=2$ gives rise to the following inequalities:

$$
\begin{aligned}
\hbar^{4} / 4+\left(G^{2,2}\right)^{2} & \leq G^{2,0} G^{2,4}+\hbar^{2}\left[3 G^{0,2} G^{2,0}-G^{2,2}\right], \\
\hbar^{4} / 4+\left(G^{2,2}\right)^{2} & \leq G^{2,0} G^{2,4}+ \\
& +\hbar^{2}\left[G^{0,2} G^{2,0}+G^{2,2}-4\left(G^{1,1}\right)^{2}\right], \\
\hbar^{4} / 4+\left(G^{2,2}\right)^{2} & \leq G^{0,4} G^{4,0}+\hbar^{2}\left[G^{2,2}-4\left(G^{1,1}\right)^{2}\right], \\
\hbar^{4} / 4+\left(G^{2,2}\right)^{2} & \leq G^{0,2} G^{4,2}+\hbar^{2}\left[3 G^{0,2} G^{2,0}-G^{2,2}\right], \\
\hbar^{4} / 4+\left(G^{2,2}\right)^{2} & \leq G^{0,2} G^{4,2}+ \\
& +\hbar^{2}\left[G^{0,2} G^{2,0}+G^{2,2}-4\left(G^{1,1}\right)^{2}\right] .
\end{aligned}
$$

Note that the first two relations (as well as the last two) are identical except for the coefficient of $\hbar^{2}$. Summing them up, it is possible to define the relation

$$
\hbar^{4} / 4+\left(G^{2,2}\right)^{2} \leq G^{2,0} G^{2,4}+2 \hbar^{2}\left[G^{2,0} G^{0,2}-\left(G^{1,1}\right)^{2}\right],
$$

where the combination of moments inside the square bracket is the same that appears in the Heisenberg uncertainty principle. The same can be done with the last couple of inequalities. Note that, as explained in Sec. IVC, the fourth classical relation derived from that inequality, $\left(C^{2,2}\right)^{2} \leq C^{4,4}$, does not give rise to a quantum uncertainty relation because the corresponding $\gamma_{n c d}$ coefficient (56) is negative and thus allows the $G^{a, b} \rightarrow 0$ limit.

The uncertainty relations at next order are the quantum analog of the classical relations (44) with $n=m=3$ :

$$
\begin{array}{lll}
\left(C^{3,3}\right)^{2} \leq C^{4,2} C^{2,4}, & & \left(C^{3,3}\right)^{2} \leq C^{6,0} C^{0,6}, \\
\left(C^{3,3}\right)^{2} \leq C^{4,0} C^{2,6}, & & \left(C^{3,3}\right)^{2} \leq C^{6,2} C^{0,4}, \\
\left(C^{3,3}\right)^{2} \leq C^{4,4} C^{2,2}, & & \left(C^{3,3}\right)^{2} \leq C^{6,4} C^{0,2}, \\
\left(C^{3,3}\right)^{2} \leq C^{4,6} C^{2,0}, & & \left(C^{3,3}\right)^{2} \leq C^{6,6} .
\end{array}
$$

All these relations are converted into quantum uncertainty relations except for $\left(C^{3,3}\right)^{2} \leq C^{4,4} C^{2,2}$ and $\left(C^{3,3}\right)^{2} \leq C^{6,6}$ that has a negative $\gamma_{n c d}$ coefficient (56) in the free term. Nonetheless, only the quantum generalization of the first two relations will be explicitly shown since the rest involve moments of an order higher than six. These are given as follows:

$$
\begin{aligned}
9 \hbar^{6} / 16+\left(G^{3,3}\right)^{2} & \leq G^{2,4} G^{4,2}+\hbar^{4}\left[3 G^{0,2} G^{2,0}-9 / 4\left(\left(G^{1,1}\right)^{2}+G^{2,2}\right)\right]+ \\
& +\hbar^{2}\left[-9 / 4\left(G^{2,2}\right)^{2}+3 G^{2,0} G^{2,4}-3 G^{1,1} G^{3,3}+G^{0,2} G^{4,2}\right], \\
\hbar^{6} / 16+\left(G^{3,3}\right)^{2} & \leq G^{2,4} G^{4,2}+\hbar^{4}\left[-9 / 4\left(G^{1,1}\right)^{2}+3 G^{0,2} G^{2,0}-1 / 4 G^{2,2}\right]+ \\
& +\hbar^{2}\left[-\left(G^{2,2}\right)^{2} / 4+3 G^{2,0} G^{2,4}-3 G^{1,1} G^{3,3}+G^{0,2} G^{4,2}\right], \\
\hbar^{6} / 16+\left(G^{3,3}\right)^{2} & \leq G^{2,4} G^{4,2}+\hbar^{4}\left[-\left(G^{1,1}\right)^{2} / 4+G^{0,2} G^{2,0}-3 / 4 G^{2,2}\right]+ \\
& +\hbar^{2}\left[-9 / 4\left(G^{2,2}\right)^{2}+G^{2,0} G^{2,4}-G^{1,1} G^{3,3}+G^{0,2} G^{4,2}\right], \\
\hbar^{6} / 16+\left(G^{3,3}\right)^{2} & \leq G^{2,4} G^{4,2}+\hbar^{4}\left[-\left(G^{1,1}\right)^{2} / 4+G^{0,2} G^{2,0}-5 / 4 G^{2,2}\right]+ \\
& +\hbar^{2}\left[-25 / 4\left(G^{2,2}\right)^{2}+G^{2,0} G^{2,4}+G^{1,1} G^{3,3}+G^{0,2} G^{4,2}\right], \\
\hbar^{6} / 16+\left(G^{3,3}\right)^{2} & \leq G^{2,4} G^{4,2}+\hbar^{4}\left[-25 / 4\left(G^{1,1}\right)^{2}+G^{0,2} G^{2,0}+7 / 4 G^{2,2}\right]+ \\
& +\hbar^{2}\left[-49 / 4\left(G^{2,2}\right)^{2}+G^{2,0} G^{2,4}+5 G^{1,1} G^{3,3}+G^{0,2} G^{4,2}\right],
\end{aligned}
$$




$$
\begin{aligned}
9 \hbar^{6} / 16+\left(G^{3,3}\right)^{2} & \leq G^{2,4} G^{4,2}+\hbar^{4}\left[3 G^{0,2} G^{2,0}-9 / 4\left(\left(G^{1,1}\right)^{2}+G^{2,2}\right)\right] \\
& +\hbar^{2}\left[-9 / 4\left(G^{2,2}\right)^{2}+G^{2,0} G^{2,4}-3 G^{1,1} G^{3,3}+3 G^{0,2} G^{4,2}\right], \\
\hbar^{6} / 16+\left(G^{3,3}\right)^{2} & \leq G^{2,4} G^{4,2}+\hbar^{4}\left[-9 / 4\left(G^{1,1}\right)^{2}+3 G^{0,2} G^{2,0}-1 / 4 G^{2,2}\right]+ \\
& +\hbar^{2}\left[-\left(G^{2,2}\right)^{2} / 4+G^{2,0} G^{2,4}-3 G^{1,1} G^{3,3}+3 G^{0,2} G^{4,2}\right], \\
\hbar^{6} / 16+\left(G^{3,3}\right)^{2} & \leq G^{2,4} G^{4,2}+\hbar^{4}\left[-9 / 4\left(G^{1,1}\right)^{2}+9 G^{0,2} G^{2,0}-1 / 4 G^{2,2}\right]+ \\
& +\hbar^{2}\left[-\left(G^{2,2}\right)^{2} / 4+3 G^{2,0} G^{2,4}-3 G^{1,1} G^{3,3}+3 G^{0,2} G^{4,2}\right], \\
9 \hbar^{6} / 16+\left(G^{3,3}\right)^{2} & \leq G^{0,6} G^{6,0}-27 / 4 \hbar^{4}\left[3\left(G^{1,1}\right)^{2}-G^{2,2}\right]+\hbar^{2}\left[-81 / 4\left(G^{2,2}\right)^{2}+9 G^{1,1} G^{3,3}\right] .
\end{aligned}
$$

[1] W. Boucher and J. H . Traschen, Phys. Rev. D 37, 3522 (1988).

[2] A. Anderson, Phys. Rev. Lett. 74, 621 (1995).

[3] T. N. Sherry and E. C. G. Sudarshan, Phys. Rev. D 18, 4580 (1978); Phys. Rev. D 20, 857 (1979).

[4] A. Peres and D. R. Terno, Phys. Rev. A 63, 022101 (2001).

[5] H. T. Elze, Phys. Rev. A 85, 052109 (2012).

[6] A. J. K. Chua, M. J. W. Hall, and C. M. Savage, Phys. Rev. A 85, 022110 (2012).

[7] J. Caro and L. L. Salcedo, Phys. Rev. A 60, 842 (1999).

[8] C. Barceló, R. Carballo-Rubio, L. J. Garay, and R. Gómez-Escalante, Phys. Rev. A 86, 042120 (2012).

[9] L. E. Ballentine, Y. Yang, and J. P. Zibin, Phys. Rev. A 50, 2854 (1994).

[10] L. E. Ballentine and S. M. McRae, Phys. Rev. A 58, 1799 (1998).

[11] M. Bojowald and A. Skirzewski, Rev. Math. Phys. 18, 713 (2006).

[12] M. Bojowald, Class. Quantum Grav. 29, 213001 (2012).

[13] M. Bojowald and A. Tsobanjan, Phys. Rev. D 80, 125008 (2009); Class. Quantum Grav. 27, 145004 (2010).

[14] M. Bojowald and R. Tavakol, Phys. Rev. D 78, 023515 (2008).

[15] M. Bojowald, D. Brizuela, H. H. Hernández, M. J. Koop, and H. A. Morales-Técotl, Phys. Rev. D 84043514
(2011).

[16] M. Bojowald, Phys. Rev. D 75, 081301 (2007); Phys. Rev. D 75123512 (2007).

[17] M. Bojowald, P. A. Höhn, and A. Tsobanjan, Class. Quantum Grav. 28, 035005 (2011); Phys. Rev. D 83, 125023 (2011).

[18] P. A. Höhn, E. Kubalova, and A. Tsobanjan, Phys. Rev. D 86, 065014 (2012).

[19] M. Bojowald, B. Sandhöfer, A. Skirzewski, and A. Tsobanjan, Rev. Math. Phys. 21, 111 (2009).

[20] D. Brizuela, in preparation.

[21] K. Banerjee, Phys. Lett. A 63, 223 (1977).

[22] J. L. Richardson and R. Blankenbecler, Phys. Rev. D 19, 496 (1979).

[23] J. B. Bronzan and R. L. Sugar, Phys. Rev. D 23, 1806 (1981).

[24] R. W. Hasse, J. Phys. A: Math. Gen. 11, 1245 (1978).

[25] B. Remaud and E. S. Hernández, J. Phys. A: Math. Gen. 132013 (1980).

[26] M. Andrews and M. Hall, J. Phys. A: Math. Gen. 18, 37 (1985).

[27] R. Glauber, Phys. Rev. 131, 2766 (1963).

[28] M. Bojowald and A. Tsobanjan, Class. Quant. Grav. 31, 115006 (2014). 